\title{
Temozolomide in combination with either veliparib or placebo in patients with relapsed-sensitive or refractory small-cell lung cancer
}

\author{
Chiara Lazzari ${ }^{1}$, Vanesa Gregorc ${ }^{1}$, Alessandra Bulotta ${ }^{1}$, Alessia Dottore ${ }^{2}$, Giuseppe Altavilla ${ }^{2}$, \\ Mariacarmela Santarpia ${ }^{2}$ \\ ${ }^{1}$ Department of Oncology, Division of Experimental Medicine, IRCCS San Raffaele Hospital, Milano, Italy; ${ }^{2}$ Medical Oncology Unit, Department \\ of Human Pathology “G. Barresi”, University of Messina, Messina, Italy \\ Correspondence to: Dr. Mariacarmela Santarpia. Medical Oncology Unit, Department of Human Pathology "G. Barresi”, University of Messina, \\ Messina, Italy. Email: msantarpia@unime.it. \\ Comments on: Pietanza MC, Waqar SN, Krug LM, et al. Randomized, Double-Blind, Phase II Study of Temozolomide in Combination With Either \\ Veliparib or Placebo in Patients With Relapsed-Sensitive or Refractory Small-Cell Lung Cancer. J Clin Oncol 2018;36:2386-94.
}

Submitted Nov 30, 2018. Accepted for publication Dec 05, 2018.

doi: $10.21037 /$ tlcr.2018.12.02

View this article at: http://dx.doi.org/10.21037/tlcr.2018.12.02

Small cell lung cancer (SCLC) accounts for approximately $15 \%$ (1) of new lung cancer cases. Conversely to the progresses in the field of non-small cell lung cancer (NSCLC), therapeutic options have not improved in the recent decades. Platinum-etoposide chemotherapy remains the cornerstone for first line treatment, while topotecan is the only approved agent for recurrent or progressive SCLC (2). Despite the initial sensitivity to chemotherapy and radiotherapy, responses are not durable, and patients develop tumor progression. Although efforts have been made to develop precision medicine approaches (3), the molecular characterization of SCLC remains poor due to the inadequate amount of tumor tissue, generally obtained by small biopsies at the time of diagnosis, and the complexity of SCLC genome, characterized by high number of somatic mutations (4). The lack of targetable actionable drivers partially explains the difficulties encountered in the development of efficient therapies, and the aggressiveness of this disease makes SCLC as a recalcitrant tumor, with a poor prognosis, and 5-year survival rate $<7 \%$ (5). Recently, a phase II study investigated the efficacy of the combination of doxorubicin with NGR-hTNF, a compound targeting angiogenesis. Results demonstrated a safety profile with a promising clinical activity (6).

The genomic instability of SCLC (7) favors the accumulation of DNA damages. SCLC tumors generally harbor bi-allelic inactivation of RB transcriptional corepressor 1 (RB1), and tumor protein p53 (TP53), involved in the regulation of G1-S cell cycle checkpoint and in DNA damage response (DDR), respectively (8). Preclinical evidences indicate that compounds targeting genes involved in DDR, as poly (ADP-ribose) polymerase (PARP) or checkpoint kinase (CHK1), might be a promising therapeutic strategy in patients with SCLC. Moreover, over expression of PARP, CHCK1/2, ataxia telangiectasia related proteins (ATR) was observed in SCLC tumors $(5,9,10)$, thus suggesting that they might be appealing targets to inhibit.

The alkylating agent temozolomide, which acts inducing DNA damages, was previously evaluated in a phase II trial, including 64 relapsed patients with SCLC, but with poor results (11). DNA repair by PARP represents one of the described resistance mechanisms to temozolomide. Based on this hypothesis, a phase II study, investigating the efficacy of temozolomide in combination with the PARP inhibitor veliparib, was conducted and recently published (12). One hundred-four patients with recurrent SCLC were enrolled. Those with brain lesions were considered eligible if asymptomatic and without leptomeningeal involvement. Patients were stratified according to the center of enrollment and the presence of a sensitive disease to previous platinum-based regimen (recurrence or progression occurring $\geq 60$ days following the end of chemotherapy), or a refractory tumor (progression or recurrence within 60 days), and randomized between temozolomide 
( $200 \mathrm{mg} / \mathrm{m}^{2} /$ day on days 1 to 5) and veliparib (40 $\mathrm{mg}$ twice daily on days 1 to 7 ) or temozolomide and placebo. The study was designed to demonstrate an improvement in terms of progression-free survival (PFS) at four months in $35 \%$ of the enrolled patients receiving the association of temozolomide and veliparib. The majority of the patients enrolled [67] had not previously received more than one line of therapy. Despite the higher objective response rate (ORR) observed in the veliparib arm, in comparison to placebo ( $39 \%$ vs. $14 \%, \mathrm{P}=0.016$ ), the primary end point of the trial was not met. No significant difference in 4-month PFS was detected between the two groups, with a median PFS of 3.8 and 2.0 months in the temozolomide/veliparib and temozolomide/placebo arms, respectively (HR 0.84; $95 \%$ CI, 0.56 to 1.25 ). The higher ORR documented in patients under veliparib was comparable between sensitive or refractory patients, and those treated with one or two prior lines of therapy. The most common adverse events reported were hematologic toxicities, especially thrombocytopenia of grade $3 / 4$, occurring in nine percent of patients in the placebo arm, and $50 \%$ of those receiving the combination. Due to hematologic toxicity, the protocol was amended, and the dose of temozolomide reduced to $150 \mathrm{mg} / \mathrm{m}^{2} /$ day. The frequent myelosuppression, treatment delays and dose reductions observed in patients receiving temozolomide/ veliparib might probably explain why ORR has not been associated with prolonged PFS. Moreover, $59 \%$ of the enrolled patients were platinum-refractory, suggesting the inclusion of a population with unfavorable clinical outcome. According to recent preclinical data, the optimal synergy between PARP-inhibitors and temozolomide may be reached administering maximal dosing of a PARP-inhibitor, while reducing that of temozolomide. In the current paper, the dose of temozolomide used was that recommended as monotherapy treatment, and was associated with a low dose of veliparib (13). All these issues might have influenced the final results.

In order to identify those patients who benefited more from the combination of temozolomide/veliparib, different biomarkers were evaluated in tissues from enrolled patients, including the presence of methylation in the $\mathrm{O}^{6}$ methylguanine-DNA methyltransferase (MGMT) gene, a well-known determinant for tumor responsiveness to temozolomide. An adequate amount of tissue was available in 32 patients only, $31 \%$ of whom carried the MGMT promoter methylated. No significant correlation was found between this biomarker and ORR, PFS or OS. Furthermore, the presence of mutations in genes involved in DDR was analyzed in 22 samples. Seven mutations in the ataxia-telangiectasia mutated $(A T M)(\mathrm{n}=5)$, breast cancer 2 (BRCA2) $(\mathrm{n}=1)$, and CHEK2 $(\mathrm{n}=1)$ genes were identified. Due to the small number, results are not conclusive. Furthermore, immunohistochemistry expression of PARP and Schlafen-11 (SLFN11) was assessed in 48 and 47 cases, respectively. While, the expression levels of PARP did not correlate with patients' outcome, SLFN11positive tumors were those with significantly prolonged PFS (5.7 vs. 3.6 months; $\mathrm{P}=0.009)$ and $\mathrm{OS}(12.2$ vs. 7.5 months; $\mathrm{P}=0.014)$ when treated with the association of temozolomide/veliparib. Conversely, no significant PFS or OS difference were observed in the temozolomide/placebo arm according to SLFN11 expression, thus confirming that SLFN11 might be a predictive marker of sensitivity to PARP-inhibition, as previously shown (14). PARPs are involved in the repair of single-strand DNA breaks (SSBs). They bind to the site of DNA damage, and induce poly(ADP-ribosyl)ation (PARylation), that is required to activate those enzymes, as the DNA ligase III or the DNA polymerase beta, that generate the base excision repair (BER) complex, implicated in DNA repair. PARPs catalyze the polymerization of ADP-ribose units from donor nicotinamide adenine dinucleotid (NAD+) molecules. PARP-inhibitors compete with NAD+, thus preventing the PARylation process, and resulting in the generation of fixed DNA-PARP complexes at SSBs, that inhibit DNA repair. Moreover, since DNA replication continues, double strand breaks are generated, leading to replication fork stalling, and as a consequence, tumor cells undergo apoptosis (5). PARP-inhibitors are particularly effective in the presence of deficiency in the homologous recombination (HR) function, or in SLFN11 positive tumors (15). Two are the hypotheses derived from preclinical studies about the role of SLFN11 in enhancing PARP-inhibitors activity. One suggests that SLFN11 removes replication protein A (RPA), a protein that prevents $\mathrm{SSB}$ and the formation of structures that would interfere with DNA repair (16). The other is that SLFN11 induces prolonged S-phase arrest. This would favor the accumulation of DNA replication, thus enhancing apoptosis, especially in the presence of PARP-inhibition, due to the generation of multiple double strand breaks and replication fork stalling (17). SLFN11 is a dynamic marker, that might undergo down-regulation following treatment with chemotherapy. Moreover, the hypermethylation of its promoter and the consequent gene silencing have been correlated to platinum resistance. Inactivation of SLFN11 has been observed in $45-50 \%$ of cancer cell lines (18). 
A

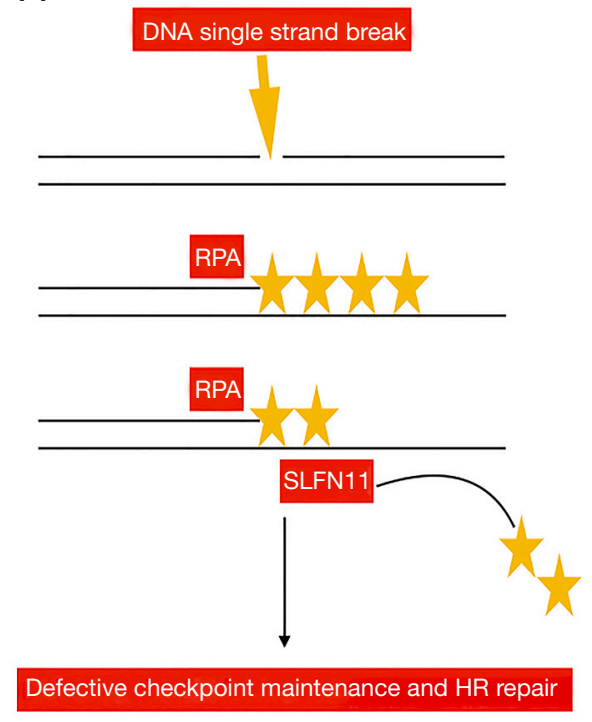

B

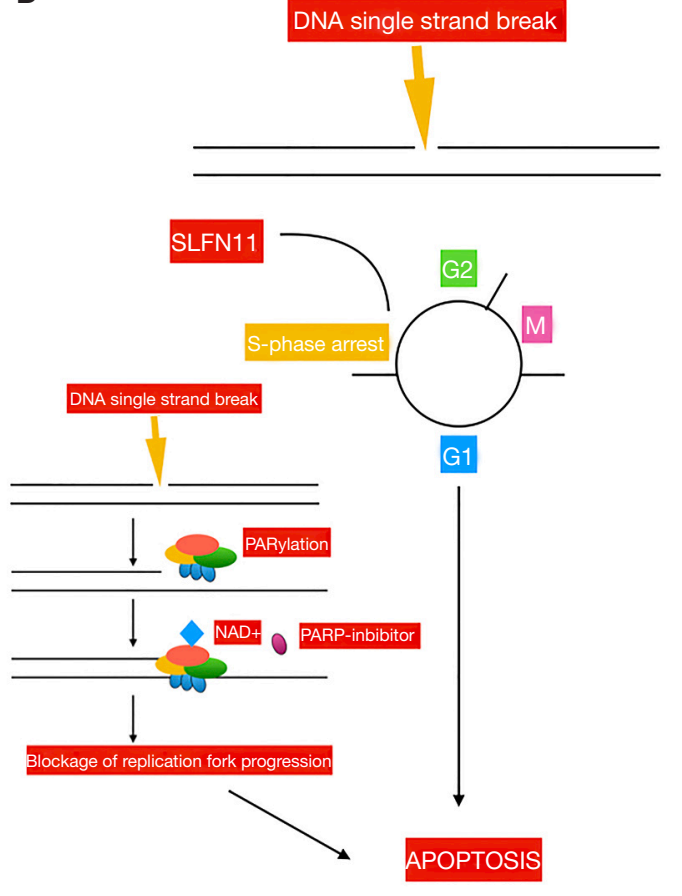

Figure 1 PARPs are involved in the repair of single-strand DNA breaks (SSBs). They bind to the site of DNA damage, and induce poly(ADP-ribosyl)ation (PARylation), that is required to activate those enzymes implicated in DNA repair. PARP-inhibitors compete with NAD+, thus preventing the PARylation process. PARP-inhibitors are particularly effective in SLFN11 positive tumors. (A) The hypothesis that SLFN11 removes replication protein A (RPA), a protein that prevents SSBs; (B) the hypothesis that SLFN11 induces prolonged S-phase arrest, thus favoring the accumulation of DNA replication, and enhancing apoptosis, especially in the presence of PARP-inhibition. Yellow stars represent RPA. PARP, poly (ADP-ribose) polymerase.

Following replicative damage, ataxia telangiectasia and Rad3-related protein kinase (ATR) phosphorylates CHK1 at serine 345, which in turn inhibits cyclin-dependent and Dbf4-dependendent kinases (CDK/DDK). CDK/DDK activates the helicase MCM2-7, which culminates in cell cycle arrest, inhibits DNA replication and promotes DNA repair (19). ATR slows cell-cycle progression, thereby allowing time for appropriate repair mechanisms to correct genetic lesions. Recently, it was shown that SLFN11 opens chromatin and inhibits fork progression regardless of ATR-CHK1 activity (19). However, in SLFN11 deficient cancer cell lines, ATR-dependent checkpoint promotes cell survival, thus suggesting that ATR inhibitors could sensitize tumor cells to PARP-inhibition in SLFN11 negative tumors (17) (Figure 1).

In conclusion, despite the combination of temozolomide/ veliparib does not improve PFS compared to temozolomide/ placebo, the study by Pietanza $e t$ al. indicates the role of SLFN11 as a predictor of sensitivity to PARP-inhibition, suggesting the promising activity of this class of compounds in molecularly selected SCLC patients. Veliparib has been explored in combination with cisplatin-etoposide in frontline setting in the ECOG-ACRIN 2511 study, with poor results (5). A phase 1/2 trial of carboplatin-etoposide with or without veliparib, using the dose of $240 \mathrm{mg}$ twice daily, and veliparib as maintenance, is currently ongoing (NCT2289690). Additional studies are investigating the PARP-inhibitor olaparib with immunotherapy. Immunotherapy has recently been introduced as a new treatment option for patients with SCLC. The activity of immune checkpoint inhibitors in SCLC has been evaluated in the phase 1/2 CA-032 study, enrolling 216 patients (20) (Table 1). Patients received nivolumab (3 $\mathrm{mg} / \mathrm{kg})$, or nivolumab $(1 \mathrm{mg} / \mathrm{kg})$ plus ipilimumab ( $1 \mathrm{mg} / \mathrm{kg})$, or nivolumab $(1 \mathrm{mg} / \mathrm{kg})$ plus ipilimumab (3 $\mathrm{mg} / \mathrm{kg})$, or nivolumab $(3 \mathrm{mg} / \mathrm{kg}$ ) plus ipilimumab $(1 \mathrm{mg} / \mathrm{kg})$. Results showed promising anti-tumor activity of these compounds. Tumor tissue was retrospectively 
Table 1 Ongoing clinical trials with PARP-inhibitors in SCLC

\begin{tabular}{lll}
\hline Trial & Inhibitor & Phase \\
\hline NCT03227016 & Veliparib + topotecan & Phase 2 \\
NCT02734004 & MEDI4736 + olaparib & Phase 1/2 \\
NCT02289690 & CBDCA + etoposide +/- veliparib & Phase 2 \\
NCT01642251 & CDDP + etoposide +/- veliparib & Phase 1/2 \\
NCT02498613 & Olaparib + cediranib & Phase 2 \\
NCT02446704 & olaparib + temozolomide & Phase 1/2 \\
NCT02511795 & Olaparib + AZD1775 & Phase 1b \\
\hline
\end{tabular}

PARP, poly (ADP-ribose) polymerase; SCLC, small cell lung cancer.

analyzed to evaluate the impact of tumor mutational burden (TMB). Greater clinical benefit was observed in those patients with high TMB (21).

Patients' selection remains of crucial importance to improve patients' prognosis in a dismal disease like SCLC. The development of effective targeted therapies requires a deeper understanding of the tumor biology. The majority of the trials investigating targeted therapies have provided disappointing results, manly because patients were not selected on the basis of the presence of specific predictive molecular alterations. The application of nextgeneration sequencing techniques in liquid biopsy might be a helpful strategy to overcome the limits related to the inadequate amount of tissue, and will probably led to a better knowledge of the molecular bases related to SCLC pathogenesis, in order to identify novel potential targets.

\section{Acknowledgements}

None.

\section{Footnote}

Conflicts of Interest: The authors have no conflicts of interest to declare.

\section{References}

1. Govindan R, Page N, Morgensztern D, et al. Changing epidemiology of small-cell lung cancer in the United States over the last 30 years: analysis of the surveillance, epidemiologic, and end results database. J Clin Oncol 2006;24:4539-44.

2. O'Brien ME, Ciuleanu TE, Tsekov H, et al. Phase III trial comparing supportive care alone with supportive care with oral topotecan in patients with relapsed small-cell lung cancer. J Clin Oncol 2006;24:5441-7.

3. Sabari JK, Lok BH, Laird JH, et al. Unravelling the biology of SCLC: implications for therapy. Nat Rev Clin Oncol 2017;14:549-61.

4. Santarpia M, Daffina MG, Karachaliou N, et al. Targeted drugs in small-cell lung cancer. Transl Lung Cancer Res 2016;5:51-70.

5. Sen T, Gay CM, Byers LA. Targeting DNA damage repair in small cell lung cancer and the biomarker landscape. Transl Lung Cancer Res 2018;7:50-68.

6. Gregorc V, Cavina R, Novello S, et al. NGR-hTNF and Doxorubicin as Second-Line Treatment of Patients with Small Cell Lung Cancer. Oncologist 2018;23:1133-e112.

7. Alexandrov LB, Nik-Zainal S, Wedge DC, et al. Signatures of mutational processes in human cancer. Nature 2013;500:415-21.

8. George J, Lim JS, Jang SJ, et al. Comprehensive genomic profiles of small cell lung cancer. Nature 2015;524:47-53.

9. Sen T, Tong P, Diao L, et al. Targeting AXL and mTOR Pathway Overcomes Primary and Acquired Resistance to WEE1 Inhibition in Small-Cell Lung Cancer. Clin Cancer Res 2017;23:6239-53.

10. Sen T, Tong P, Stewart CA, et al. CHK1 Inhibition in Small-Cell Lung Cancer Produces Single-Agent Activity in Biomarker-Defined Disease Subsets and Combination Activity with Cisplatin or Olaparib. Cancer Res 2017;77:3870-84.

11. Pietanza MC, Kadota K, Huberman K, et al. Phase II trial of temozolomide in patients with relapsed sensitive or refractory small cell lung cancer, with assessment of methylguanine-DNA methyltransferase as a potential biomarker. Clin Cancer Res 2012;18:1138-45. 
12. Pietanza MC, Waqar SN, Krug LM, et al. Randomized, Double-Blind, Phase II Study of Temozolomide in Combination With Either Veliparib or Placebo in Patients With Relapsed-Sensitive or Refractory Small-Cell Lung Cancer. J Clin Oncol 2018;36:2386-94.

13. Smith MA, Reynolds CP, Kang MH, et al. Synergistic activity of PARP inhibition by talazoparib (BMN 673) with temozolomide in pediatric cancer models in the pediatric preclinical testing program. Clin Cancer Res 2015;21:819-32.

14. Polley E, Kunkel M, Evans D, et al. Small Cell Lung Cancer Screen of Oncology Drugs, Investigational Agents, and Gene and microRNA Expression. J Natl Cancer Inst 2016;108.

15. Lok BH, Gardner EE, Schneeberger VE, et al. PARP Inhibitor Activity Correlates with SLFN11 Expression and Demonstrates Synergy with Temozolomide in Small Cell Lung Cancer. Clin Cancer Res 2017;23:523-35.

16. Mu Y, Lou J, Srivastava M, et al. SLFN11 inhibits

Cite this article as: Lazzari C, Gregorc V, Bulotta A, Dottore A, Altavilla G, Santarpia M. Temozolomide in combination with either veliparib or placebo in patients with relapsed-sensitive or refractory small-cell lung cancer. Transl Lung Cancer Res 2018;7(Suppl 4):S329-S333. doi: 10.21037/tlcr.2018.12.02 checkpoint maintenance and homologous recombination repair. EMBO Rep 2016;17:94-109.

17. Murai J, Feng Y, Yu GK, et al. Resistance to PARP inhibitors by SLFN11 inactivation can be overcome by ATR inhibition. Oncotarget 2016;7:76534-50.

18. Barretina J, Caponigro G, Stransky N, et al. The Cancer Cell Line Encyclopedia enables predictive modelling of anticancer drug sensitivity. Nature 2012;483:603-7.

19. Murai J, Tang SW, Leo E, et al. SLFN11 Blocks Stressed Replication Forks Independently of ATR. Mol Cell 2018;69:371-84.e6.

20. Antonia SJ, Lopez-Martin JA, Bendell J, et al. Nivolumab alone and nivolumab plus ipilimumab in recurrent smallcell lung cancer (CheckMate 032): a multicentre, openlabel, phase 1/2 trial. Lancet Oncol 2016;17:883-95.

21. Hellmann MD, Callahan MK, Awad MM, et al. Tumor Mutational Burden and Efficacy of Nivolumab Monotherapy and in Combination with Ipilimumab in Small-Cell Lung Cancer. Cancer Cell 2018;33:853-61.e4. 